\title{
Discovery of a stellar system in the background of 47 Tucanae
}

\section{A new cluster of the Small Magellanic Cloud?}

\author{
M. Bellazzini ${ }^{1}$, E. Pancino ${ }^{1}$, and F. R. Ferraro ${ }^{2}$ \\ 1 INAF - Osservatorio Astronomico di Bologna via Ranzani 1, 40127, Bologna, Italy \\ e-mail: [michele.bellazzini; elena.pancino]@bo.astro.it \\ 2 Università di Bologna, Dipartimento di Astronomia, via Ranzani 1, 40127, Bologna, Italy \\ e-mail: francesco.ferraro3@unibo.it
}

Received 14 December 2004 / Accepted 6 February 2005

\begin{abstract}
We report on the discovery of a stellar system in the background of the Galactic globular cluster 47 Tucanae (NGC 104), located $14.8^{\prime}$ North-West of the cluster center. The object, whose apparent diameter is $D \simeq 30^{\prime \prime}$, is partially resolved into stars on the available CCD images, reaching a limiting magnitude of $V \sim 22.5$, and is detected as a significant (more than $5 \sigma$ ) overdensity of blue stars $(B-V<0.7)$. The color magnitude diagram of the system, its characteristic projected size and its position in the sky suggest that it is an intermediate-old age cluster belonging to the Small Magellanic Cloud, whose outskirts lie in the background of 47 Tuc. Although less likely, the possibility that the object is an unknown dwarf galaxy in the outskirts of the Local Group cannot completely be ruled out by the present data.
\end{abstract}

Key words. Galaxy: globular clusters: individual: Bo A - galaxies: Local Group

\section{Introduction}

The advent of modern surveys exploring large areas of the sky at different wavelenghts, that provide deeper insight into poorly-explored regions of the Galaxy and its surroundings, has lead to a burst of discoveries and cataloguing of new stellar systems and structures that has no precedent in the past three decades. For example, the insight into high extinction regions provided by infrared imaging (2MASS, Spitzer) has allowed the recognition of several stellar systems that were hidden into the Galactic disc, or in general, in low galactic latitude fields (see, for example Bica et al. 2003a,b; Kobulnicky et al. 2004).

Also, well known high-surface-brightness objects subtending large solid angles in the sky, such as some nearby globular clusters or for instance M 31, may hide behind their body resolved stellar systems such as star clusters or dwarf galaxies (see Whiting et al. 2000). Here we briefly report on the curious case of a stellar system we discovered in the background of the nearby globular cluster 47 Tucanae.

\section{Observations}

Observations were obtained at the $2.2 \mathrm{~m}$ ESO-MPI telescope at ESO (La Silla) in July 1999 with the Wide Field Imager

* Based on data collected at the European Southern Observatory, La Silla, Chile, with the Wide Field Imager under the observing programmes 62.L-0354 and 64.L-0439. Also based on Wide Field Imager data retrieved from the ESO/ST-ECF Science Archive Facility.
(WFI) and an additional dataset was also retrieved from the ESO/ST-ECF Science Archive. The final set consisted of several $B, V$ and $I$ images (six or seven $60 \mathrm{~s}$ exposures in each filter) centered on the globular cluster 47 Tucanae. Details of the data reduction procedure and other results obtained from this dataset can be found in Ferraro et al. (2004) and Bellazzini et al. (2004).

The WFI is a mosaic of eight $2048 \times 4096$ pixel chips with a scale of $0.238^{\prime \prime}$ pixel $^{-1}$. The whole mosaic covers an area of $\simeq 34^{\prime} \times 33^{\prime}$. During the visual inspection of the images we noted, on chip 4 of the $B$ and $V$ frames, an unusual bright spot. A closer look revealed the presence of an anomalous clustering of faint stars, placed over a barely visible concentration of unresolved light. By averaging the three best seeing $B$ images (chip 4 only), we obtained the combined image shown in Fig. 1, revealing that we have found a partially resolved stellar system, in the background of the much closer cluster 47 Tuc. For brevity we named it Bologna A (Bo A).

\section{The nature of Bo A}

As can be noticed from Fig. 1, the overall shape of Bo A is roundish and the apparent diameter is $\sim 30^{\prime \prime}$. The position of the optical center of symmetry is RA $=0^{\mathrm{h}} 21^{\mathrm{m}} 30.5^{\mathrm{s}}$ and Dec $=-71^{\circ} 56^{\prime} 7^{\prime \prime}$ (equinox 2000.0). We searched the NED, SIMBAD and Vizier databases as well as other databases (such as the catalogue of SMC clusters by Bica \& Dutra (2000), hereafter BD00) and we found no known object within $2^{\prime}$ of this 


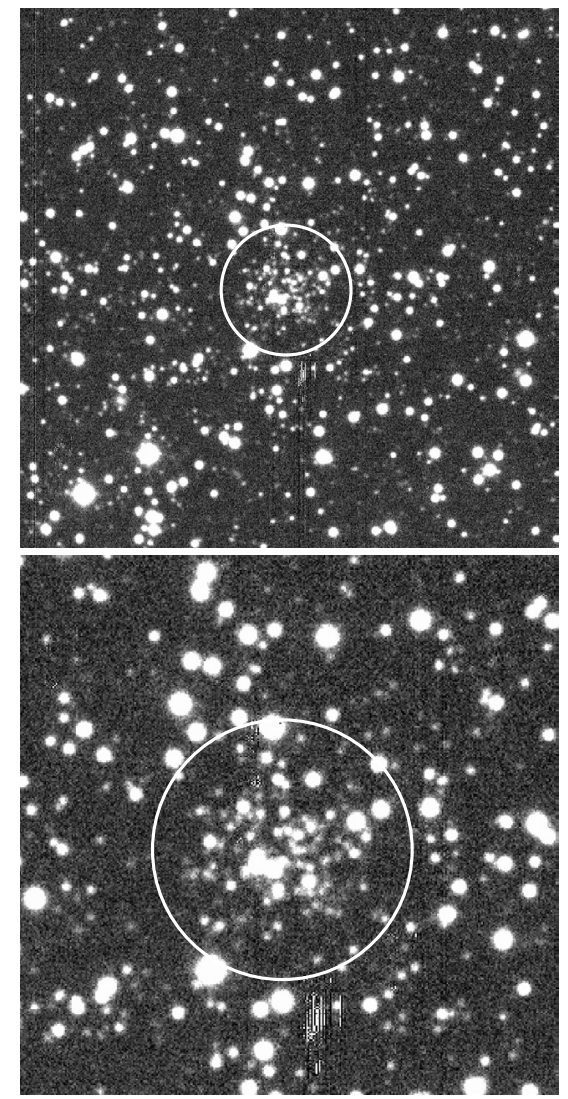

Fig. 1. Upper panel: combined $B$ image of $2.7^{\prime} \times 2.7^{\prime}$ centered on the newly discovered stellar system. North is up and East is left. Lower panel: the same region zoomed in by a factor of $\sim 2$. The overplotted circles have a diameter of $40^{\prime \prime}$. Virtually all the bright stars present in the image are members of the globular cluster 47 Tucanae.

position, except for a few bright stars belonging to 47 Tuc. Bo A is clearly visible in all of our $B$ and $V$ images but is hard to recognise in $I$ images, suggesting that it is dominated by blue stars. It is also (barely) visible in the DSS-2 images.

In the following we discuss the properties and nature of Bo A as can be deduced from our dataset.

\subsection{Position in the sky}

It is well known that 47 Tuc is projected onto the outskirts of the Small Magellanic Cloud (SMC), a galaxy which has a rich cluster system (469 confirmed members, according to BD00). Figure 2 shows the sky-projected map of the confirmed SMC clusters from the catalogue of BD00 (open circles). The center (Djorgovski \& Meylan 1993) and tidal radius (Trager et al. 1993) of 47 Tuc are marked by a cross and a large circle, respectively. Bo A is represented by the filled square: clearly, its position is fully compatible with that of a SMC cluster. Hence, the most natural hypothesis is that Bo A is a previously unknown SMC cluster, projected by chance near 47 Tuc. The postition of Bo A can explain why it has never been identified before, lying only just $\simeq 14.8^{\prime}$ away from the center of this cluster. The previous record of proximity to 47 Tuc was held by HW5 (Hodge \& Wright 1974), located 34.6' away from the center.

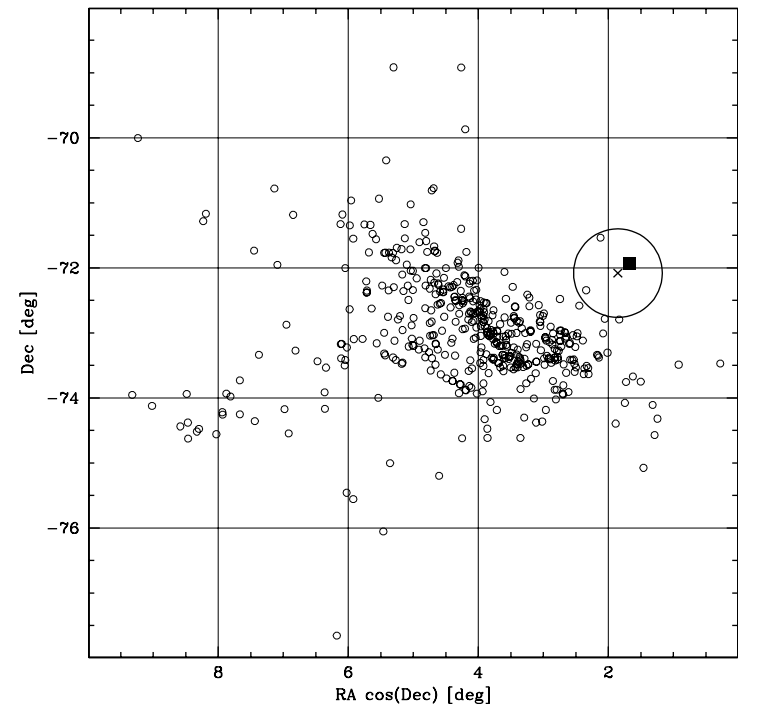

Fig. 2. Projected map of all the confirmed SMC clusters from the catalogue by Bica \& Dutra (2000) (open circles). The cross marks the position of the center of 47 Tuc, the circle has the radius equal to its tidal radius. Bo A is represented by the filled square.

\subsection{Color magnitude diagram}

Since the previous published photometric analysis of this dataset was aimed at obtaining the cleanest possibile sample of stars in 47 Tuc (Bellazzini et al. 2004; Ferraro et al. 2004), only stars that were detected in at least three images per passband were included in the final catalogues. Here we noted that the stellar population of Bo A appears faint and blue, therefore it is not clearly visible in the shallow exposures, and it is barely visible on the $I$ band images. Therefore, we took the best seeing $B$ and $V$ images of chip $4\left(F W H M=0.8^{\prime \prime}\right)$ and performed new photometry with less restrictive requirements, to reach as deep as possible.

The resulting Color Magnitude Diagram (CMD) of Bo A is shown in Fig. 3 (left panel), where we plotted only stars within $1^{\prime}$ of the center of Bo A (small dots). Stars lying within the apparent radius of Bo A $\left(r<15^{\prime \prime}\right)$ are highlighted as large filled circles. The CMD is dominated by the Main Sequence (MS) of 47 Tuc (going approximately from $V \sim 17, B-V \simeq 0.8$ to $V \sim 22, B-V \simeq 1.6$ ), while most of the stars within the apparent body of Bo A appear quite faint $(V>20.0)$ and blue $(B-V<0.8)$.

\subsection{Bo $A$ as an overdensity of blue stars}

We now use the CMD of Fig. 3 to assess the statistical significance of Bo A as an overdensity of resolved stars with respect to the underlying field of 47 Tuc. There are 38 stars with $V>20.0$ lying within $r \leq 15^{\prime \prime}$ from the center of Bo A. To study the probability of a chance occurrence of such a clustering, we counted the total number of faint $(V>20.0)$ stars $\left(N_{\mathrm{F}}\right)$ in 10000 circles, having $r=15^{\prime \prime}$, randomly placed on chip 4 . We added the further condition that their centers avoid a region 


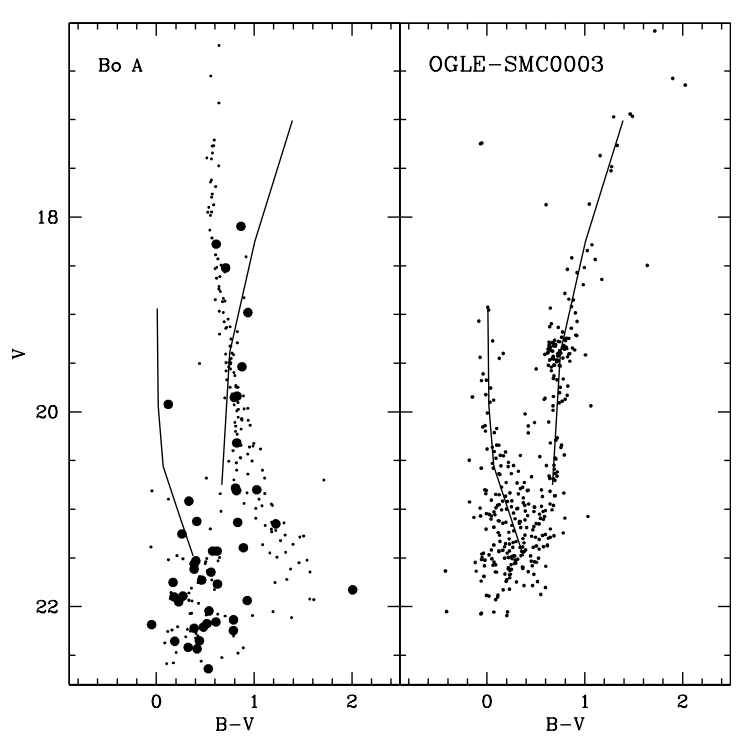

Fig. 3. Left panel: CMD of all the stars within $r=1^{\prime}$ from the center of Bo A (tiny dots). Stars with $r \leq 15^{\prime \prime}$ are highlighted (large filled circles). The thin sequence of stars going from $V \sim 17, B-V \simeq$ 0.8 to $V \sim 22, B-V \simeq 1.6$ is the MS of 47 Tuc. The ridge lines of the upper MS and of the RGB of OGLE-SMC0003 (Pietrzynski et al. 1998) are overplotted on both panels. Right panel: CMD of the Small Magellanic Cloud cluster OGLE-SMC0003 from Pietrzynski et al. (1998).

of radius $r=2^{\prime}$ from the center of Bo $\mathrm{A}^{1}$. The average over the 10000 random extractions is $\left\langle N_{\mathrm{F}}\right\rangle=9.7 \pm 3.1$ (Poisson statistics), therefore $N_{\mathrm{F}}=38$ measured in Bo A is significantly different at the $5.5 \sigma$ level. This is graphically illustrated in the top left panel of Fig. 4, where it can also be appreciated that $N_{\mathrm{F}}$ values equal to or higher than 38 happen in $0.1 \%$ of the cases only. Note that the adopted Poisson statistics provides an error that is fully compatible with the observed distribution.

Moreover, as we already noted, Bo A appears bluer than the surrounding field, with 27 out of 38 faint stars bluer than $B-V \leq 0.7$. We therefore repeated the experiment, this time counting the number of faint $(V>20.0)$ and blue $(B-V \leq 0.7)$ stars $\left(N_{B}\right)$ in 10000 circles of $r=15^{\prime \prime}$, randomly placed on chip 4 as above. The average over 10000 random extractions is $\left\langle N_{B}\right\rangle=1.7 \pm 1.3$ (Poisson statistics), where the $N_{B}=27$ measured for Bo A is significantly different, at the $5 \sigma$ level. The result is illustrated in the top right panel of Fig. 4, where it can be noted that the maximum value obtained for $N_{B}$ is only 8 .

Finally, if we consider the ratio of faint blue stars to faint red stars $N_{B} / N_{R}$ (where faint red stars are those having $B-V>$ 0.7 and $V>20.0$ ) we obtain $\left\langle N_{B} / N_{R}\right\rangle=0.1 \pm 1.3$ over 10000 random extractions, to compare with the observed value of $N_{B} / N_{R}=2.45 \pm 0.88$ for Bo A. Therefore, we can conclude not only that Bo A is a statistically significant aggregation of resolved stars in the field of 47 Tuc, but also that its stellar population differs from that encountered anywhere else in chip 4, being significantly bluer.

${ }^{1}$ We recall here that the field covered by chip 4 is $\simeq 8.1^{\prime} \times 16.2^{\prime}$ and that it samples the outer halo of 47 Tuc, hence the crowding is moderate all over the chip, and finally Bo A is not located where the density of stars of 47 Tuc is the highest.
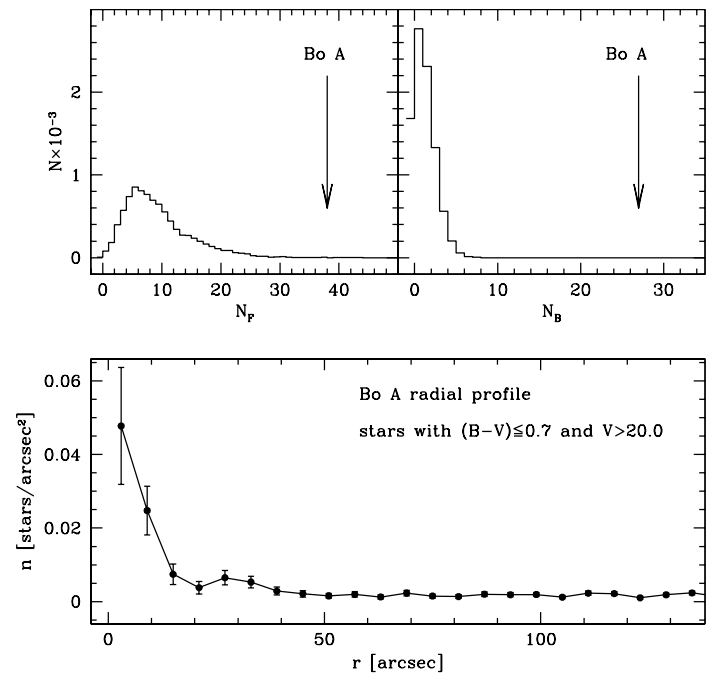

Fig. 4. Upper left panel: stars counted in 10000 randomly placed circles of radius $15^{\prime \prime}$ in the field around Bo A, having $V>20.0$. The number of stars counted in the circle centered on Bo A $\left(N_{\mathrm{F}}=38\right)$ is marked by the arrow. Upper right: same as above, but only for blue stars, having $V>20.0$ and $B-V \leq 0.7$. The number counted in the circle around Bo A $\left(N_{B}=27\right)$ is marked by the arrow. Lower panel: radial density profile of Bo A, obtained using faint blue stars $(V>20.0$ and $B-V \leq 0.7)$ and $6^{\prime \prime}$ bins. The profile flattens outside $r \sim 20^{\prime \prime}$ and remains flat over the whole chip 4 field (not shown here for reasons of scale).

\subsection{Shape, size and density profile}

The density contours map of blue stars has been computed on circles of radius $r=10^{\prime \prime}$, placed on a grid spaced by $5^{\prime \prime}$. The only structure emerging above the $5 \sigma$ level on the background is Bo A, shown in Fig. 5, and the peak density is more than $16 \sigma$ higher than the background. A radial density profile has also been computed in 6" bins, using the faint blue stars as tracers of Bo A (Fig. 4, lower panel). It can be readily appreciated that the density of these stars becomes essentially flat outside $\sim 20^{\prime \prime}$ from the center of the system and remains flat out to the edges of the considered field.

Thus, Bo A appears as a quite concentrated, approximately circular overdensity, with a characteristic apparent diameter of $D \simeq 30^{\prime \prime}$ (see Figs. 1 and 4). We note that according to BD00 the typical diameters of confirmed SMC clusters range from $\geq 12^{\prime \prime}$ to $\sim 4^{\prime}$, where $25 \%$ of the whole sample has $D \leq 30^{\prime \prime}$. Hence, the characteristic size of Bo A is fully compatible with the hypothesis that it is a cluster of the SMC galaxy. In this hypothesis, and assuming a distance modulus of $(m-M)_{0}=$ 18.82 (Mateo 1998) for the SMC, the linear size of Bo A would therefore be of approximately $8.4 \mathrm{pc}$.

\subsection{Stellar content}

Now that we have established the statistical significance of Bo A as an aggregate of resolved stars, we can use the CDM of Fig. 3 to study its stellar content. The ridge lines of the upper MS (the blue plume at $B-V<0.6)$ and of the Red Giant Branch (RGB, going from $V \sim 21$ and $B-V \sim 0.8$ to $V \sim 17$ and $B-V \sim 1.4$ ) of OGLE-SMC0003 are overplotted to both 


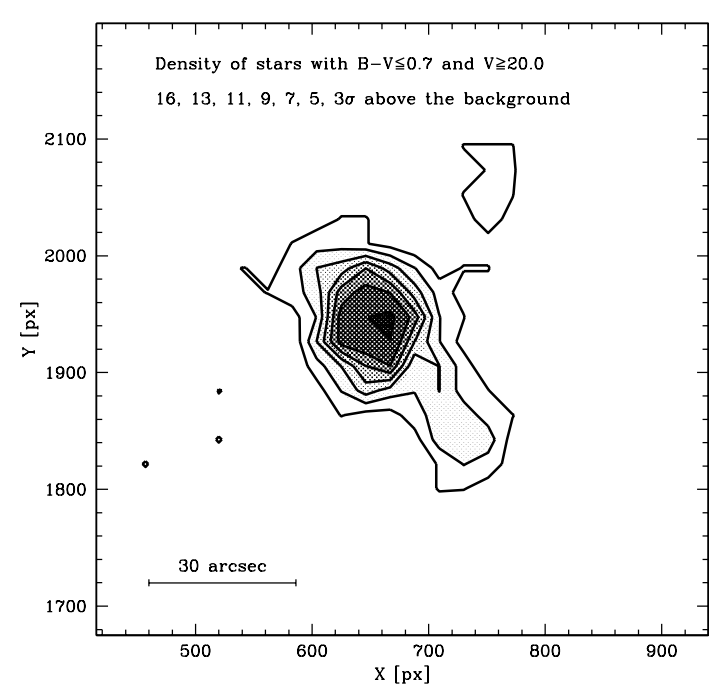

Fig. 5. $2^{\prime} \times 2^{\prime}$ cutout of the density map of faint blue stars $(V>20.0$ and $B-V<0.7$ ) on chip 4. The map is computed by counting stars in circles of $r=10^{\prime \prime}$, placed on a grid spaced by $5^{\prime \prime}$. The average density (computed over the whole chip) has been subtracted and the remaining over-density has been divided by the standard deviation of the background, hence it is expressed in units of $\sigma$. Only around Bo A the density of stars reaches a level of $5 \sigma$ above the background, while the peak density is more than $16 \sigma$.

CMDs in Fig. 3. We can conclude from this comparison that the CMD of Bo A is fully compatible with that of a sparse cluster belonging to the SMC. In this case the plume of blue stars, lying around the extrapolation of the template MS ridge line at faint magnitudes, should be interpreted as the upper MS of the cluster, while most of the redder stars may belong to its RGB.

The lower luminosity of the bright end of the MS could imply that the age of Bo A is larger than that of OGLE-SMC0003 ${ }^{2}$, although small statistics prevent us from reaching a firm conclusion on this point. The presence of a fully developed RGB and of a well-defined Red Clump would imply that OGLE-SMC0003 is older than 2-3 Gyr, which can provide a tentative lower limit to the age of Bo A as well. However, it has to be noted that the putative RGB stars of Bo A are still fully compatible in color, magnitude and number with being MS stars of 47 Tuc. There are in fact 8 stars within $15^{\prime \prime}$ of the center of Bo A with $0.4<B-V<1.1$ and $V<20.5$. In a set of 10000 random extractions such as those described above, 8 or more stars with these characteristics are found in $20 \%$ of the cases, hence there is no significant overdensity of RGB stars around Bo A. A fainter MS with respect to OGLE-SMC0003, coupled with the lack of a corresponding RGB would argue against the hypothesis that Bo A is an ordinary SMC cluster and may instead suggest that is a much farther system (this possibility is briefly considered in Sect. 4 , below). In any case, low number statistics prevent us from making any firmer statement, at the present stage.

It is clear that a better characterization of the stellar content of Bo A requires a much deeper and higher resolution photometry. We therefore searched various scientific archives for better

\footnotetext{
${ }^{2}$ In the hypothesis that the two clusters were approximately at the same disctance.
}

images of a field including Bo A, but the only promising dataset (a set of $t_{\text {exp }}=900 \mathrm{~s}$ images taken with the ESO-NTT) was not useful because of the very bad seeing under which they were acquired $\left(F W H M \geq 2.5^{\prime \prime}\right)$. Hence, dedicated observations are needed for a deeper insight.

\section{Conclusions}

We have identified a previously unknown stellar system (Bo A) in the background of the galactic globular cluster 47 Tucanae, located $\sim 15^{\prime}$ North-West of its center. The system, which we call Bo A, is partially resolved into stars and is clearly detected as a statistically significant overdensity of faint and blue stars in the considered $\simeq 8.1^{\prime} \times 16.2^{\prime}$ field (chip 4), located in the outskirts of 47 Tuc. The appearance, the characteristic size, the CMD and the position in the sky of Bo A indicate that it is most probably a stellar cluster belonging to the Small Magellanic Cloud. The proximity of Bo A to 47 Tuc is the most likely explanation of why the system has not been discovered before.

While the identification of Bo A as a SMC cluster is most likely, the available data (and, in particular, the shallow CMD) leave formally open (at least) another interpretation (see Sect. 3.4, above). A dwarf irregular galaxy located at $\sim 1 \mathrm{Mpc}$ or more from us (like, for instance, Sag DIG Lee \& Kim 2000) may appear similar to Bo A if seen in the background of 47 Tuc, whose diffuse brightness may hide the unresolved body of the galaxy. Its CMD would appear very similar to that of Bo A, once the same limiting magnitude $(V \sim 22.5)$ is attained (see, Fig. 2 of Lee \& Kim 2000). Hence, accurate photometry down to $V \sim 24$ is required to definitely rule out this less likely, but still viable, hypothesis.

Acknowledgements. Financial support for this research has been provided by the Agenzia Spaziale Italiana (ASI) and the Ministero dell'Istruzione, dell'Università e della Ricerca (MIUR). We are grateful to the anonymous Referee for her/his detailed and useful suggestions.

\section{References}

Bellazzini, M., Ferraro, F. R., Sollima, A., Pancino, E., \& Origlia, L. 2004, A\&A, 424, 199

Bica, E., \& Dutra, C. M. 2000, AJ, 119, 1214 (BD00)

Bica, E., Dutra, C. M., Soares, J., \& Barbuy, B. 2003a, A\&A, 400, 533

Bica, E., Dutra, C. M., Soares, J., \& Barbuy, B. 2003b, A\&A, 404, 217

Djorgovski, S., \& Meylan, G. 1993, in Structure and Dynamics of Globular Clusters, ed. S. Djorgovski, \& G. Meylan, ASP Conf. Ser., 50, 325

Ferraro, F. R., Beccari, G., Rood, R. T., et al. 2004, ApJ, 603, 127

Hodge, P. W., \& Wright, F. W. 1974, AJ, 79, 858

Kobulnicky, C., Monson, A. J., Buckalew, B. A., et al. AJ, in press [arXiv: astro-ph/0410400]

Lee, M.-G., \& Kim, S.-C. 2000, AJ, 119, 777

Mateo, M. L. 1998, ARA\&A, 36, 435

Pietrzynski, G., Udalski, A., Kubiak, M., et al. 1998, Acta Astron., 48, 175

Trager, S., Djorgovski, S., \& King, I. R. 1993, in Structure and Dynamics of Globular Clusters, ed. S. Djorgovski, \& G. Meylan, ASP Conf. Ser., 50, 373

Whiting, A. B., Hau, J. T. K., \& Irwin, M. 2000, ApJS, 141, 123 